\title{
Analysis on the Development Trend of E-commerce Live Streaming
}

\author{
Fengju Hou* \\ Zibo Vocational Institute, Zibo, Shandong 255000, China.
}

\begin{abstract}
Based on the current development status of e-commerce live broadcasting in China, this paper predicts the development prospects of e-commerce live broadcasting on the basis of analyzing the successful elements, advantages and disadvantages of e-commerce live broadcasting. This paper points out that e-commerce live broadcasting will become the "standard configuration" of e-commerce, brand and business in the future, which can continue to develop continuously.
\end{abstract}

Keywords: E-commerce Live Broadcasting; Development Trend; E-commerce Live Streaming; PrivateTraffic; Influencers

\section{Current situation of e-commerce live streaming}

E-commerce live streaming, also known as live broadcasting, is a new mode of economic. Although it has existed in China for several years, it developed rapidly in 2019, which is known as the first year of e-commerce live streaming. On Singles Day Shopping Festival of 2019, Taobao's live streaming platform generated \$2.85 billion in sales streaming. On continuously. adv's total sales. Taobao'aobaol streaming platform is both an early adopter and leader in the e-commerce live streaming space.

The epidemic situation in 2020 changed people's consumption concept and demand, China's e-commerce live broadcasting industry will continue to maintain the growth trend.

\section{Advantages and disadvantages of e-commerce live broadcasting}

\subsection{Advantages}

Compared with pure pictures and short videos, live broadcast can let consumers see all aspects of the goods more directly. The real-time language and emotion of the host and the real-time feedback of the audience will make the goods more real and reduce the cost of trust ${ }^{[2]}$. It also can make users perceive their own services, users' demands can get corresponding quickly, and the streamer can also get users' feedback quickly.

Live broadcasting of e-commerce helps to break the bottleneck of e-commerce platform in finding traffic. It also improves the shopping experience, especially the stars can attract popular traffic, realize precision marketing.

\subsection{Disadvantages}

Live usually cannot see the details of the product, touch its texture, and cannot accurately perceive the product. The lighting and lens of the live broadcasting room have a great influence on the appearance of goods, which may mislead consumers easy to produce impulse consumption, which will lead to more returns in the later period and increase the after-sales cost.

Copyright (C) 2020 Fengju Hou

doi: $10.18282 / 1-e . v 9 i 4.1724$

This is an open-access article distributed under the terms of the Creative Commons Attribution Non-Commercial License (http://creativecommons.org/licenses/by-nc/4.0/), which permits unrestricted non-commercial use, distribution, and reproduction in any medium, provided the original work is properly cited.

[7]Song-Iee H, Leslie H, Sharon B. Structural Relationships Between Social Activities and Longitudinal Trajectories of Depression Among Older Adults[J]. The Gerontologist, 2009, 49 (1): 1.

[8]Jang Y, Mortimer J A, Haley W E, et al. The role of social engagement in life satisfaction: Its significance among older individuals with disease and disability[J]. Journal of Applied Gerontology, 2004, 23(3): 266-278.

[9] Wang Lili. A review of the theory, empirical and Policy Research on the social participation of the elderly in China [J]. Population \& Development, 2011, 17(3): 35-43.

[10] Arens D A. Widowhood and well-being: An examination of sex differences within a causal model[J]. The International Journal of Aging and Human Development, 1983, 15(1): 27-40.

[11] FOLLAND S. Does D Snternational Journal l"Does D Snternational Journal of A[J]. Social Science and Medicine, 2007, $64(11): 2342-2354$.

[12] Jang, Y., Haley, W. E., \& Graves, A. M. The role of social engagement in late satisfaction: Its significance among older individuals with disease and disability[J]. The Journal of Applied Gerontology, 2004, 23(3), 266-278.

[13] Xie Lili, Wang Bin. Social Participation Profile of the Chinese Elderly in the Context of Active Ageing: Patterns and Determinants [J]. Population Research, 2019, 43(03): 17-30. 


\section{Elements for successful live broadcasting of e-commerce}

\subsection{Platform or private traffic}

E-commerce giants, represented by Taobao and Jingdong, are trying to introduce new traffic through live broadcast, precipitate content, promote transformation and stimulate consumption

The content platform represented by Douyin and Kuaishou, are trying to expand its business channel through broadening its revenue channels.

And offline retail enterprises (commercial circulation companies represented by Suning) are also trying to reconstruct traffic interaction channels and optimize trading links through live broadcast, linking online and offline scenes.

Right now, the super app WeChat is the biggest private traffic channel in China. Brands are using personal WeChat accounts and WeChat groups to create communities of consumers.

\subsection{Streamer or host}

When live streaming e-commerce first appeared, it was delivery by the host who specialized in network live broadcast. Such as Li Jiaqi of Taobao. However, with the development of live e-commerce and the impact of the epidemic this year, the types of streamer continue to be rich, and the number of host also continues to grow. Streamer in different fields, such as e-sports, food and entertainment, have also added a delivery link in their live broadcast.

With a mobile phone and a selfie stick, farmers can display fresh fruits and vegetables in their greenhouses, and small selfemployed farmers can introduce the performance of their products in their factories. There are also a large number of stars and brand managers, and even government officials have joined the ranks of live broadcasting, becoming the main force of diversified live broadcasting e-commerce.

Rely on the personal charm of the streamer to attract fans, most host

focus on meeting the needs of users and solving users' pain points.

\subsection{Products}

For factories, it is well known that online traffic is expensive and customer acquisition cost is high. E-commerce live mode breaks through the bottleneck of customer acquisition transformation, saves the steps of innovation, promotion and retention, directly sells goods, and opens up the sales volume of products, which is very meaningful for the initial brand building of products.

\section{Development trend of e-commerce live streaming ${ }^{[3]}$}

Live broadcasting will become the standard configuration for e-commerce, brands and businesses. With the rapid increase of live broadcasting penetration rate and the catalysis of epidemic situation, live broadcasting and delivery will enter the "era of the whole people". Live broadcast e-commerce, influencers are becoming a new growth point, and the penetration rate is also increasing rapidly.

It can be predicted that live broadcast will become the "standard configuration" of e-commerce, brands and businesses in the future, and can continue to develop.

Live broadcast can be regarded as a kind of ROI advertising method. In the future, VR, AI and robotics are the future trends, which can be combined with live broadcast to continuously improve the user experience. It will develop in the direction of high quality and high quality, showing new trends such as increasing varieties and diversified consumption.

\section{Reference}

[1]Wu Xiaobo channel. E-commerce live broadcast War: inner volume is the ultimate destiny of the Internet stock age. [EBOL]. https://ishare.ifeng.com/mediaShare/home/323/media

[2]Financial information. The new pattern of live e-commerce and the era of diversification have arrived.[EBOL]. http:// www.51emo.com/caijing/2021/0106/012021_29052.html.

[3]How to explain these Chinese expressions in English.

http://language.chinadaily.com.cn/a/202001/18/WS5e225893a310128217271d7b.html.China Daily 2020-01-18 09:00 